\title{
Endoscopic Ultrasound-Guided Transgastric Puncture and Drainage of an Adrenal Abscess in an Immunosuppressed Patient
}

\author{
Carlos Andrés Regino', Jean Paul Gómez', and Gabriel Mosquera-Klinger ${ }^{2}$ \\ ${ }^{1}$ Internal medicine, University of Antioquia, Medellin, ${ }^{2}$ Gastroenterology and Endoscopy Unit, Pablo Tobon Uribe Hospital, Medellin, \\ Colombia
}

\begin{abstract}
Adrenal gland infection is a clinical entity of great importance, but it is a largely unrecognized pathology. Immunosuppressed individuals are at a higher risk of presentation. Herein, we describe a young female patient, recently diagnosed with HIV, who presented with severe sepsis due to methicillin-resistant Staphylococcus aureus, associated with a left adrenal abscess. She was initially treated with antibiotics; however, due to the persistence of the systemic inflammatory response and bacteremia, endoscopic ultrasound-guided drainage was performed. This procedure was successful in resolving the clinical situation.

Endoscopic ultrasound-guided adrenal gland drainage can be a safe, efficacious, and minimally invasive option for managing antibiotic-refractory adrenal abscesses in immunosuppressed patients. Clin Endosc 2022;55:302-304
\end{abstract}

Key Words: Abscess; Adrenal glands; Drainage; Endoscopic ultrasound-guided fine-needle aspiration; Endosonography

\section{INTRODUCTION}

Adrenal gland infection is an important but largely unrecognized clinical entity. It can be caused by a wide variety of pathogens that include fungi, viruses, parasites, and bacteria. ${ }^{1}$ Immunocompromised individuals are at a higher risk for primary infection of the adrenal glands or infection due to disseminated disease. ${ }^{1}$ Standard treatment of intra-abdominal abscesses, including adrenal abscesses, currently consists of antibiotic therapy and percutaneous image-guided drainage techniques, such as ultrasound or tomography. ${ }^{2}$ However, percutaneous drainage can be difficult due to the interposition of the liver, lung, pleura, or intestinal segments, making surgical drainage necessary. Nevertheless, surgical drainage is an inva-

\footnotetext{
Received: February 27, 2021 Revised: April 18, 2021

Accepted: April 19, 2021

Correspondence: Gabriel Mosquera-Klinger

Calle 78B \#69-240, private office 154, Pablo Tobon Uribe Hospital, Medellin, Colombia

Tel: +57-3147920257, Fax: +57-3147920257, E-mail: gami8203@yahoo.com ORCID: https://orcid.org/0000-0002-3108-0424
}

(cc) This is an Open Access article distributed under the terms of the Creative Commons Attribution Non-Commercial License (http://creativecommons.org/ licenses/by-nc/3.0) which permits unrestricted non-commercial use, distribution, and reproduction in any medium, provided the original work is properly cited. sive procedure that should be avoided in immunosuppressed patients presenting with infections, in patients with multiple comorbidities, and in patients who are hemodynamically unstable, given that it could increase morbidity and mortality. ${ }^{3}$ In selected cases, the interventional technique of endoscopic ultrasound (EUS) enables the puncture and drainage of collections in patients in whom percutaneous treatment is difficult or unfeasible. ${ }^{4}$

\section{CASE REPORT}

A 15-year-old female, with a history of recurrent furunculosis since infancy, arrived at the emergency department because of abdominal pain in the epigastrium and left hypochondrium with a 2-month progression, associated with nausea and vomiting. One week earlier, she presented with a spiking fever and pronounced malaise. Upon admission, the patient's general condition was poor. Physical examination identified low blood pressure, tachycardia, and abdominal tenderness, with no signs of peritoneal irritation, and oral cavity examination revealed whitish plaques, consistent with oropharyngeal candidiasis. The patient was transferred to the special care unit, where crystalloid resuscitation and vasopressor support with norepi- 

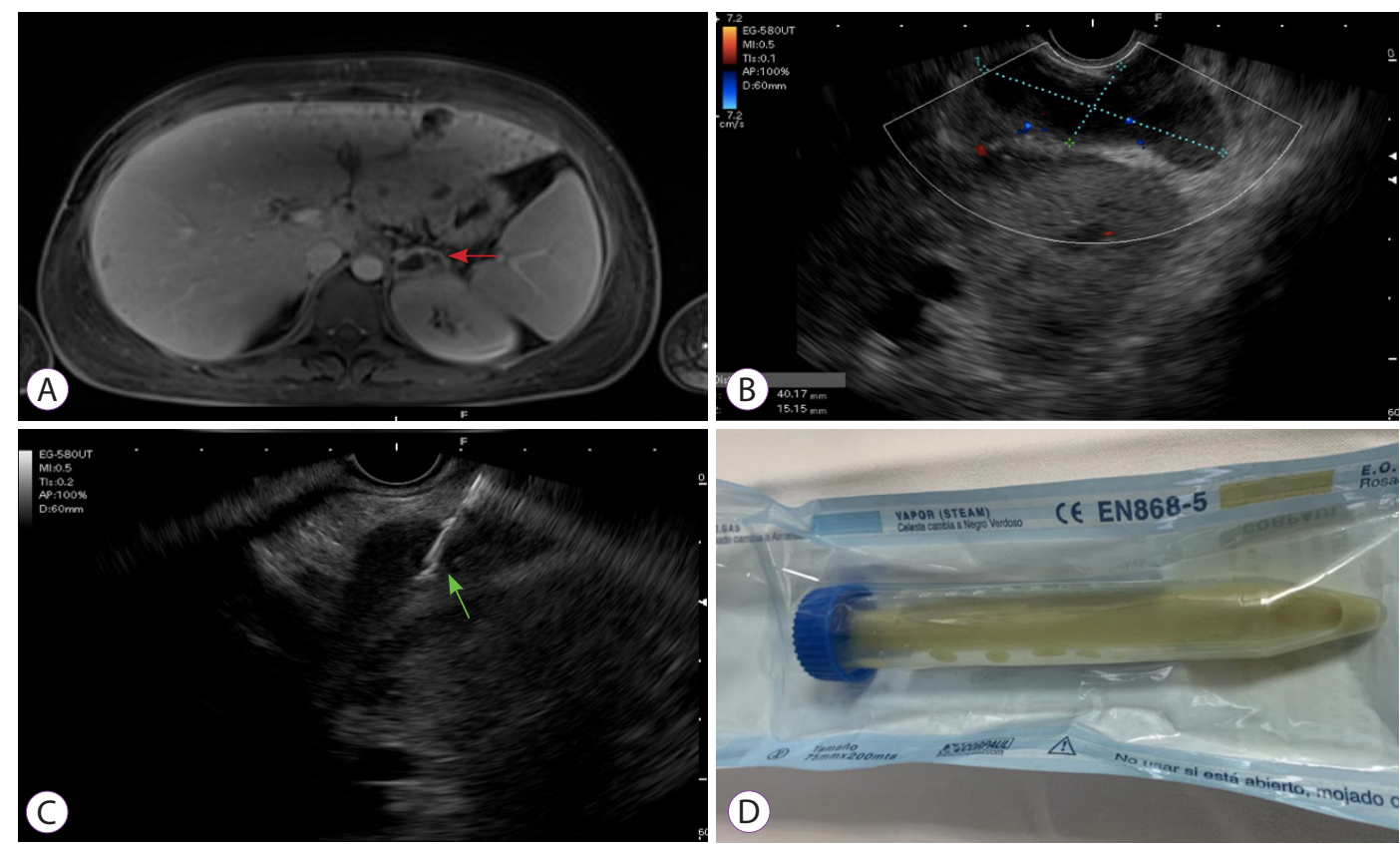

Fig. 1. (A) Abdominal magnetic resonance imaging, axial section, showing wall enhancement in the left adrenal gland, with evidence of fluid content (red arrow). (B) Linear endoscopic ultrasound view, identifying the markedly enlarged left adrenal gland, with content that appears to be mixed (anechoic/hypoechoic). (C) Aspiration puncture/drainage of the content (green arrow). (D) A portion of the extracted purulent material sent to the microbiology unit in a dry tube.

nephrine were initiated. The initial tests reported normocytic normochromic anemia, lymphopenia, mild thrombocytopenia, elevated C-reactive protein $(27 \mathrm{mg} / \mathrm{dL})$, hyperlactatemia, and compromised liver function, with ALT 357 U/L, AST 309 $\mathrm{U} / \mathrm{L}$, and hyperbilirubinemia. Taking the physical examination findings and the initial laboratory test abnormalities into account, an ELISA HIV test was performed. The result was positive, with an elevated viral load (63,626 copies) and a CD4 lymphocyte count of 32 . Due to the marked inflammatory response, blood cultures were carried out and antibiotic therapy with vancomycin and meropenem was empirically initiated. An abdominal ultrasound study identified hepatosplenomegaly and free fluid in the lesser sac. Complementary contrast-enhanced abdominal magnetic resonance imaging showed multiple round millimetric liver lesions consistent with micro-abscesses, along with findings in the left adrenal gland consistent with a collection that was not amenable to percutaneous drainage (Fig. 1A). Methicillin-resistant Staphylococcus aureus (MRSA) growth was observed in blood cultures. After 72 hours of treatment with intravenous antibiotics, the symptoms persisted with signs of systemic inflammatory response and positive blood cultures. Infectious endocarditis was ruled out through transesophageal echocardiogram and, given that there were no other collections amenable to drainage, the decision was made to drain the abscess in the left adrenal gland.

Endoscopic ultrasonography was performed with the patient under general anesthesia. Using a linear endoscope, the transgastric puncture was made with a $19 \mathrm{G}$ needle (Expect needle $^{\mathrm{TM}}$; Boston Scientific, Marlborough, MA, USA), and 20 $\mathrm{mL}$ of pus was sent for microbiological analysis. The area was washed out with the same quantity of saline solution at $0.9 \%$ until the drainage was clear (Fig. 1B-D).

After drainage, the general status of the patient improved with modulation of the inflammatory response. Vasopressor support was suspended 2 days after drainage, and the control blood cultures were negative. The patient was discharged 1 week after the transgastric drainage, and she completed a 6-week outpatient parenteral antimicrobial drug therapy with vancomycin. The cultures obtained from the purulent drained fluid were positive for MRSA, ruling out other opportunistic infections, such as tuberculosis, fungi, and parasites. Follow-up was done during the outpatient parenteral treatment and 12 weeks after discharge with no symptom relapse. Due to the successful clinical outcomes, we did not consider follow-up images after aspiration. 


\section{DISCUSSION}

Adrenal glands can be compromised in HIV patients through several routes, including direct infection, opportunistic infection at that level, and alterations related to anti-retroviral medications. The most frequent causes of adrenal involvement in HIV are cytomegalovirus, Mycobacterium tuberculosis, Cryptococcus neoformans, Histoplasma capsulatum, Pneumocystis jirovecii, and Toxoplasma gondii, which are opportunistic pathogens that are usually associated with adrenal insufficiency. On the other hand, bacteria can affect the adrenal glands by direct infection and tissue damage or by exotoxin and endotoxin production. ${ }^{1}$ Waterhouse-Friderichsen syndrome is described as a rapidly progressing entity, in which sepsis induces bilateral adrenal hemorrhage. The germs related to sepsis and compromised adrenal glands are $N$. meningitidis, $H$. influenza, pneumococcus, $P$. multocida, $K$. oxytoca, and $S$. aureus, among others, but are rarely isolated in the adrenal glands in autopsies of these patients. ${ }^{1}$

In general, intra-abdominal abscesses tend to be severe infectious entities. The treatment included long-term antibiotic therapy and drainage to control the inoculum. Regarding adrenal gland infections, simple percutaneous drainage through aspiration, especially in pediatric populations, has been described with good clinical results in case reports. ${ }^{5-7}$ In the subphrenic location, the percutaneous approach continues to be the method of choice, but it is known that those collections can be near the lungs, pleura, liver, and intestines, which can affect visualization or make drainage risky. ${ }^{3,8,9}$ In this context, the use of minimally invasive methods, such as EUS, can be of diagnostic value in determining the cause, as well as being therapeutic through puncture and aspiration. We found no cases of EUS-guided puncture and drainage of adrenal abscesses in our review of the literature. In our case, we underlined the fact that we performed the puncture with drainage and washout, with no need for a permanent catheter in place.

On the other hand, the patient met all three criteria for outpatient parenteral antimicrobial drug therapy implementation. The patient had an active infectious disease that required continued treatment beyond the anticipated period of hospitalization. Other than the treatment of the infectious disease, there was no need for further hospitalization. Finally, there were no equally effective and safe oral antimicrobial regimens. ${ }^{10}$

We conclude that adrenal gland infections are still not widely recognized, but the possible benefits of their early recognition and intervention, especially when they involve fungal pathogens or bacteria, justify having a high clinical suspicion, especially in systemic infections. ${ }^{11}$ Simple EUS-guided drainage of the adrenal gland is less invasive and can be safer in immunosuppressed individuals than in other drainage methods.

\section{Conflicts of Interest}

The authors have no potential conflicts of interest.

Funding

None.

Author Contributions

Conceptualization: Carlos Andrés Regino, Jean Paul Gómez, Gabriel Mosquera-Klinger

Data curation: CAR, JPG, GMK

Visualization: CAR, JPG, GMK

Writing-original draft: CAR, JPG, GMK

Writing-review \& editing: CAR, JPG, GMK

ORCID

Carlos Andrés Regino Jean Paul Gómez

https://orcid.org/0000-0003-3187-5478 Gabriel Mosquera-Klinger

https://orcid.org/0000-0003-2202-6378 https://orcid.org/0000-0002-3108-0424

\section{REFERENCES}

1. Paolo WF, Nosanchuk JD. Adrenal infections. International Journal of Infectious Diseases 2006;10:343-353.

2. Mehta N, Ii EC. Abdominal Abscess. [Internet]. Treasure Island (FL); StatPearls; c2020 [updated 2020 Jun 23; cited 2021 Apr 22]. Available from: https://www.statpearls.com/ArticleLibrary/viewarticle/17019.

3. Politano AD, Hranjec T, Rosenberger LH, Sawyer RG, Tache Leon CA. Differences in morbidity and mortality with percutaneous versus open surgical drainage of postoperative intra-abdominal infections: a review of 686 cases. Am Surg 2011;77:862-867.

4. Sooklal S, Chahal P. Endoscopic Ultrasound. Surg Clin North Am 2020;100:1133-1150.

5. Yokoyama S, Sekioka A, Utsunomiya H, Hara S, Takahashi T, Yoshida A. Adrenal abscess as a complication of Escherichia coli sepsis in neonates: A case report. J Ped Surg Case Reports 2013;1:328-330.

6. Diepstraten SCE, Zwaveling S, Beek FJA. Diagnosis and subsequent US-guided percutaneous drainage of an adrenal abscess in a 5-week-old infant. Pediatr Radiol 2012;42:1126-1129.

7. Mandelia A, Kishore JS, Yadav R, Lal R. Non-surgical management of bilateral adrenal abscess in neonates: report of two cases. J Neonatal Surg 2017;6:31.

8. Morita S, Kamimura K, Suda T, et al. Endoscopic ultrasound-guided transmural drainage for subphrenic abscess: report of two cases and a literature review. BMC Gastroenterol 2018;18:55.

9. Prasad GA, Varadarajulu S. Endoscopic ultrasound-guided abscess drainage. Gastrointest Endosc Clin N Am 2012;22:281-290, ix.

10. Gilbert DN, Dworkin RJ, Raber SR, Leggett JE. Outpatient parenteral antimicrobial-drug therapy. N Engl J Med 1997;337:829-839.

11. Patil R, Ona MA, Papafragkakis C, Duddempudi S, Anand S, Jamil LH. Endoscopic ultrasound-guided fine-needle aspiration in the diagnosis of adrenal lesions. Ann Gastroenterol 2016;29:307-311. 